\title{
The chemical chaperon 4-phenylbutric acid improves cardiac function following isoproterenol-induced myocardial infarction in the rat
}

\section{Fatemeh Vatankhah}

Tabriz University of Medical Sciences Faculty of Pharmacy

\section{Alireza Garjani}

Tabriz University of Medical Sciences Faculty of Pharmacy

Haleh Vaez ( $\square$ haleh.vaez@gmail.com )

Tabriz University of Medical Sciences Faculty of Pharmacy https://orcid.org/0000-0001-5331-1441

\section{Research Article}

Keywords: 4-phenylbutric acid, Isoproterenol, Myocardial infarction, Autophagy, Chaperon

Posted Date: February 16th, 2022

DOl: https://doi.org/10.21203/rs.3.rs-1249891/v1

License: (c) (i) This work is licensed under a Creative Commons Attribution 4.0 International License. Read Full License 


\section{Abstract}

Purpose 4-phenyl butyric acid (4-PBA) is a chaperone-mediated autophagy (CMA) inducer, which eliminates unnecessary and damaged cellular components through lysosomal enzymes. It could reduce misfolded and unfolded proteins produced after myocardial infarction (MI) and can improve cardiac function. We aimed to investigate the effect of 4-PBA on isoproterenol-induced $\mathrm{Ml}$ in the rat.

Methods Isoproterenol (100 mg/kg) was injected subcutaneously for two consecutive days simultaneous with an intraperitoneal (IP) injection of 4-PBA at 20, 40, or $80 \mathrm{mg} / \mathrm{kg}$ with 24-h intervals for five days. On day 6 , hemodynamic parameters, histopathological changes, peripheral neutrophil count, and total antioxidant capacity (TAC) evaluated. The expression of autophagy proteins measured by using western blotting. 4-PBA significantly improved post-MI changes in hemodynamic parameters.

Results Histological improvement found in 4-PBA $40 \mathrm{mg} / \mathrm{kg}(\mathrm{P}<0.05)$. The neutrophils count in the peripheral blood significantly decreased in the treatment groups compared with the isoproterenol. Furthermore, 4-PBA at $80 \mathrm{mg} / \mathrm{kg}$ significantly increased the serum TAC compared to isoproterenol $(P<0.001)$. Western blotting results showed a significant decrease in the P62 level $(P<0.05)$ of 40 and $80 \mathrm{mg} / \mathrm{kg} 4-\mathrm{PBA}$ treated groups.

Conclusion This study demonstrated that 4-PBA could have a cardio-protective effect against isoproterenol-induced $\mathrm{MI}$, which can be due to the modulation of autophagy and inhibition of oxidative stress. Obtaining effective results in different doses shows the need of optimum degree of authophagic activities in cells.

\section{Introduction}

Myocardial ischemia is among the most important causes of morbidity and mortality worldwide, with a $42 \%$ increased prevalence in recent years [1]. Myocardial ischemia occurs due to an obstruction of coronary arteries and consequently decreased blood flow to the coronary arteries, leading to myocardial infarction (MI). Tissue remodeling, necrosis, fibrosis, hypertrophy, inflammation, and accumulation of neutrophils at the site of infarction and subsequently increased destruction due to the activity of proteolytic enzymes are among the consequences of MI [2].

Physiological stresses such as oxidative stress occur in MI, causing several changes in cells, and affecting the structure and function of proteins. In this process, unfolded and misfolded proteins also synthesized, which can cause further cardiac damage and apoptosis of cardiac cells. These proteins activate the unfolded protein response pathway, which can cause further tissue damages [3,4]. Autophagy is an intracellular pathway that helps to eliminate the unfolded and misfolded proteins and plays a critical catabolic role for cell survival against different stress types such as the endoplasmic reticulum stress $[3,5]$. The process of autophagy can activate programmed cell death under specific circumstances, and serves as an alternative to the apoptotic pathway [6]. Autophagy is divided into three main types of macro-autophagy, micro-autophagy, and chaperone-mediated autophagy (CMA) [7]. Microautophagy is mediated by lysosomal action through a direct engulfment of the cytoplasmic cargo. In macro-autophagy, an autophagosome with a bilayer membrane is formed and engulfs the damaged organelles and the unusable proteins. It then delivers them to a vacuole to destroy and discard them [8-10]. CMA is a selective form of autophagy in mammals. In this process, some specific proteins containing the KFERQ amino-acid sequence are detected by the chaperones and unfolded. Subsequently, they are delivered into the lysosomes by lysosome-associated membrane protein type 2A and degraded [11]. Macroautophagy and CMA, both maximally switched on in response to stress. As the sequential activation of these two pathways, cross-talk between these stress-related autophagic pathways are investigated in different studies [12]. 
The effective proteins in the macro-autophagy pathway include the Beclin1, P62, and LC3. By measuring the level of these proteins, the autophagic activity of the cells can be quantified [13]. Evidence shows that Beclin1 regulates autophagy and membrane trafficking involved in physiological and pathological processes [14]. P62 identifies the intracellular residues, and takes the role in their elimination. Impairment of autophagy would lead to accumulation of P62 in cells, leading to cellular stress and eventual disease $[15,16]$. Thus, an increased level of this protein indicates decreased autophagy $[17,18]$. It was reported that blockage of CMA activates macroautophagy in cultured cells [19]. Furthermore, a bidirectional cross-talk between macroautophagy and CMA was established which means changes in the activity of one of these pathways will affect the protein breakdown contribution of the other pathway [20].

In the process of autophagy, the cytoplasmic form of LC3 (LC3-I) is converted to conjugated LC3 (LC3-II), which is absorbed by the autophagosomal membrane. Since LC3-II within the autophagosomal membrane is degraded by the lysosomal hydrolase, the transient amount of LC3-II cannot indicate autophagic activity. Thus, LC3-II lysosomal turnover is estimated to determine the autophagic activity [21, 22]. The GAPDH protein is used as a standard along with the aforementioned three proteins to assess their relative amount [23].

When autophagy moderately augmented, it exerts protective effects on cell function, such as ATP production and clearance of oxidized proteins and injured organelles. On the other hand, excessive activation of autophagy is associated with the risk of eliminating of essential proteins and intracellular organelles, which can cause cell death [24].

Chemical chaperones induce the CMA pathway, as the ability of mimicking the function of chaperones in the autophagy pathway. They enhance the transfer of mutated and misfolded proteins and improve the folding capacity of the endoplasmic reticulum [25]. The 4-phenyl butyric acid (4-PBA) is a non-toxic chemical chaperone and a type of fatty acid with low molecular weight. The American Food and Drug Administration has approved the application of 4PBA [26], and is clinically used as an ammonia scavenger in children with urea cycle disorder and the treatment of sickle cell anemia and thalassemia [27]. It has maximum uptake by the heart and kidneys and was recently manufactured in controlled release form due to its short half-life [28, 29]. Previous in vivo studies have documented the potential efficacy of 4-PBA in reducing the complications due to ischemia-reperfusion, fibrosis, and cardiac necrosis in rats $[30,31]$. There is a gap in existing knowledge in the area of effects of chemical chaperones such as 4PBA on MI. Thus, this in vivo study aimed to assess the role of 4-PBA on hemodynamic and histopathological factors following induction of MI by isoproterenol in the rats. In addition, proteins involved in autophagy such as Beclin1, LCII/I, and P62 are evaluated to further understanding the compensatory mechanisms between the autophagic pathways in $\mathrm{MI}$.

\section{Materials And Methods}

\section{Chemical reagents}

Isoproterenol was purchased (Sigma Chemicals, USA), 4-PBA (Merck, Germany), Ketamin and (Alfasan, Netherland), Xylazine (Alfasan, Netherland), Acepromazine (Alfasan, Netherland) and Formaldehyde (Merck, Germany) were used in this study. The other reagents were of a commercial analytical grade.

\section{Experimental animals}

In this study, a total of 30 male Wistar rats weighing 270-300 g were selected and randomly assigned to six standard propylene cages ( $\mathrm{n}=5$ in each cage). The rats were housed at $25-27^{\circ} \mathrm{C}$ and $50 \pm 10 \%$ humidity under $12 \mathrm{~h} \mathrm{light} / 12 \mathrm{~h}$ dark cycles with ad libitum access to food and water. The technician taking care of the rats was blinded to the study 
objectives. In case of the appearance of wound, infection, or death of a rat during the study period, it was excluded from the study and replaced with another rat.

This study was conducted under the institutional guidelines for the care and use of animal models. The approvement by the ethics committee of Tabriz University of Medical Sciences obtained (IR.TBZMED.VCR.REC.1397.139 and IR.TBZMED.VCR.REC.1397.048). In addition, the study designed and implemented under the recommendations of the ARRIVE guidelines for reporting animal research [32].

\section{Experimental procedures}

The rats randomly divided into five groups $(n=6)$ : healthy control, isoproterenol, and three doses of 4-PBA (P20, P40, and P80) using a simple randomization software (Microsoft Excel 2016; Microsoft Co., Redmond, WA, USA).

The healthy control rats received twice-subcutaneous injection of $0.3 \mathrm{ml}$ saline at the back of their neck with a 24-h interval. In addition, they received five injections of saline $(0.3 \mathrm{ml}, \mathrm{IP})$ with $24-\mathrm{h}$ intervals. In the isoproterenol group to induce Ml, isoproterenol dissolved in saline at a dosage of $100 \mathrm{mg} / \mathrm{kg}$ and subcutaneously injected into the back of rat's neck (in two consecutive days with a 24-h interval). In addition, similar to the control group, saline (0.3 $\mathrm{ml}$, IP) was injected daily for five days starting from the first day.

The rats in groups P20, P40, and P80 received five intraperitoneal injections of 4-PBA at a dosage of 20,40, and 80 $\mathrm{mg} / \mathrm{kg}$, respectively. Simultaneously, all 4-PBA groups received two injections of isoproterenol as the same of isoproterenol group. The surgical procedure was performed $96 \mathrm{~h}$ after the second isoproterenol injection. $\mathrm{NaOH}$ was used for better dissolution of 4-PBA and the $\mathrm{pH}$ was adjusted at 7.4 [33]. The addition of $\mathrm{NaOH}$ converted the weak acid of 4-PBA, to sodium salt, similar to the saline group.

The rats in all five groups were anesthetized by intraperitoneal injection of ketamine, xylazine and acepromazine. Afterward rats underwent a surgical procedure on day 6 (96 h after the second isoproterenol injection).

\section{Measurement of hemodynamic parameters}

The open chest technique was used to assess the left ventricle function and direct measurement of left ventricle pressure alterations. For this purpose, the chest was opened and a catheter was directly inserted into the left ventricle. The pressure change at a fixed ventricular pressure (LV dP/dt/P), left ventricular maximal and minimal rates of pressure increase $\left(\mathrm{LV} d P / d t_{\min }\right.$ and $\left.L V d P / d t_{\text {max }}\right)$, left ventricular systolic pressure (LVSP) and left ventricular enddiastolic pressure (LVEDP), mean arterial pressure (MAP) and heart rate (HR) were recorded [34, 35].

\section{Blood collection and preparing the slides}

Once the abdomen of the rats was opened, the blood was collected from the hepatic portal vein using a 10-ml syringe. One drop of blood was placed on a slide and spread. After drying, it was fixed by dripping methanol over it, and stained by Giemsa stain (Merck). The peripheral blood neutrophils were evaluated under a microscope (Olympus, Japan) at x100 magnification. The remaining blood was stored in the blood collecting tubes and plasma was separated from the blood cells by cold centrifugation (Eppendorf AG, Germany) at 3200 rpm for 20 min.

\section{Cardiac examination}

After opening the chest, the heart was removed and rinsed with cold saline at $4^{\circ} \mathrm{C}$ and weighed. To assess cardiac hypertrophy and edema, the ratio of the heart weight (in grams) to the rat body weight (in kilograms) was calculated. The cardiac tissue of 15 rats were frozen at $-70^{\circ} \mathrm{C}$ for western blotting and the others were fixed in $10 \%$ formalin for histopathological studies. Fixed tissues, embedded in paraffin, and sectioned into pieces with 5 - $\mu$ m thickness. The 
specimens were stained with hematoxylin and eosin (Sigma Aldrich, USA) and inspected under a microscope at x40 magnification. Cardiac fibrosis and necrosis were assessed in each section by the morphometric point-counting procedure [36]. A blinded technician scored the histopathological changes as mild (score 1), moderate (score 2), severe (score 3), or very severe (score 4).

\section{Measuring the serum TAC}

The serum TAC was measured in plasma samples and assessed using the Ferric reducing ability of plasma (FRAP) method [37]. The method is based on the principle of the reduction of the ferric-tripyridyltriazine complex to the ferrous form by the antioxidants of a sample, which causes the development of an intense blue color, which is spectrophotometrically measurable at $593 \mathrm{~nm}$ and the change in absorbance is related to the antioxidant capacity of sample.

\section{Western blotting}

A total of $40 \mathrm{mg} / \mathrm{kg}$ of the cardiac tissue apex was dissected and lysed in $2 \mathrm{ml}$ of the lysis buffer using a homogenizer. Then centrifuged at $1000 \mathrm{rpm}$ at $4^{\circ} \mathrm{C}$ for 10 minutes and the supernatant was stored at $-70^{\circ} \mathrm{C}$ for further analysis. For western blotting, first, total protein was quantified by NanoDrop (Thermo, USA). Next, 50-100 $\mu \mathrm{g}$ protein was removed from the solution and electrophoretically transferred onto a polyvinylidene fluoride membrane using SDS-PAGE (12\%). The membranes were blocked with $6 \%$ nonfat dried milk in phosphate-buffered saline (pH of 7.2) for one hour at room temperature. After blocking the non-specific binding sites, the membranes were rinsed with the wash buffer and placed in a solution containing primary antibodies, and incubated overnight at $4^{\circ} \mathrm{C}$. The primary antibodies included the rabbit polyclonal anti-Beclin1 (1:1000; Cell Signaling, USA), rabbit polyclonal anti-P62 (1:1000; Cell Signaling, USA), rabbit polyclonal anti-LC3 (1:1000; Cell Signaling, USA), and mouse polyclonal antiGAPDH (1:2500; Abcam, England). Next, all membranes were rinsed with the wash buffer and incubated at room temperature for one hour in a solution containing secondary antibodies such as goat anti-rabbit lgG horseradish peroxidase-conjugated secondary antibody (1:2000; Cell Signaling, USA) for Beclin1, P62, and LC3, and goat antimouse IgG horseradish peroxidase-conjugated secondary antibody (1:5000; Abcam, England) for GAPDH. The proteins were detected by the chemiluminescence method, and the appeared bands were observed by an imaging device. The optical density of the bands was quantified by an image scanning analysis software (Image j; Wayne Rasband, National Institute of Health, USA) and reported as optical density per square millimeter.

\section{Statistical analysis}

Data were analyzed using SPSS version 25 (SPSS Inc., IL, USA). The data were presented as mean \pm standard error of the mean (SEM). ANOVA was used to compare the data among the study groups. In case the presence of a significant difference, the Fisher's least significant difference (LSD) test was applied. Differences between groups were considered significant if $p<0.05$.

\section{Results}

\section{Hemodynamic parameters}

As demonstrated in Table 1, isoproterenol made a significant decrease in the MAP and LVSP $(P<0.01$ and $P<0.001$ respectively versus the healthy control group). MAP in $\mathrm{P} 20$ and $\mathrm{P} 40$ groups were significantly higher $(\mathrm{P}<0.001, \mathrm{P}<0.01$ respectively) compared to the isoproterenol. In the same way, 4-PBA significantly augmented the LVSP in the P20 and P40 groups $(P<0.001)$. 4-PBA in concentration of $80 \mathrm{mg} / \mathrm{kg}$ could make no significant difference in MAP and LVSP. 
Table 1

Effects of 4-PBA on hemodynamic parameters and left ventricular function

\begin{tabular}{|c|c|c|c|c|c|c|c|}
\hline Groups & $\begin{array}{l}\text { MAP } \\
(\mathrm{mmHg})\end{array}$ & $\begin{array}{l}\text { HR } \\
\text { (bpm) }\end{array}$ & $\begin{array}{l}\text { LVSP } \\
(\mathrm{mmHg})\end{array}$ & $\begin{array}{l}\text { LVEDP } \\
(\mathrm{mmHg})\end{array}$ & $\begin{array}{l}\mathrm{LV} \\
\mathrm{dP} / \mathrm{dt} / \mathrm{P} \\
(1 / \mathrm{sec})\end{array}$ & $\begin{array}{l}\mathrm{LV} \mathrm{dP} / \mathrm{dt}_{\max } \\
(\mathrm{mmHg} / \mathrm{sec})\end{array}$ & $\begin{array}{l}\mathrm{LV} \mathrm{dP} / \mathrm{dt}_{\min } \\
(\mathrm{mmHg} / \mathrm{sec})\end{array}$ \\
\hline Control & $74 \pm 8.5$ & $275.1 \pm 5.7$ & $71.6 \pm 3.7$ & $2.6 \pm 0.8$ & $4.9 \pm 0.6$ & $1146 \pm 91$ & $-964 \pm 168$ \\
\hline Iso & $49.2 \pm 6.2^{\star *}$ & $217 \pm 6.3^{\star \star}$ & $35.6 \pm 2.9^{\star \star \star}$ & $12.6 \pm 1.5^{\star \star \star}$ & $1.2 \pm 0.3^{\star \star \star}$ & $582 \pm 117^{* *}$ & $-546 \pm 159^{*}$ \\
\hline P20 & $87.2 \pm 4.8^{\# \# \#}$ & $217.5 \pm 8.6$ & $77 \pm 1.9^{\# \# \#}$ & $5.4 \pm 1 \# \#$ & $4 \pm 0.4^{\# \#}$ & $1146 \pm 106^{\# \#}$ & $-1039 \pm 121^{\#}$ \\
\hline P40 & $76.1 \pm 3.9^{\# \#}$ & $235.6 \pm 10.5$ & $79.5 \pm 5.9^{\# \# \#}$ & $4.6 \pm 0.8^{\# \# \#}$ & $3.1 \pm 0.4^{\#}$ & $952.5 \pm 131^{\#}$ & $-835 \pm 165$ \\
\hline P80 & $46.2 \pm 4.5$ & $212.24 \pm 25.1$ & $43.67 \pm 3$ & $7.4 \pm 1.4^{\# \#}$ & $1.8 \pm 0.7$ & $440 \pm 52$ & $-356.5 \pm 49$ \\
\hline \multicolumn{8}{|c|}{ 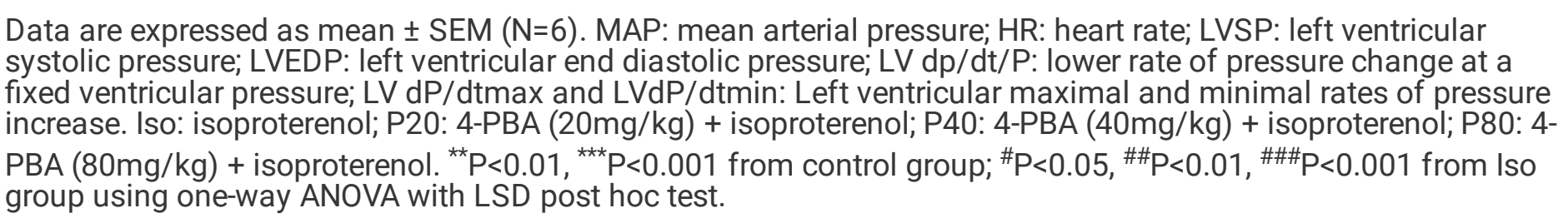 } \\
\hline
\end{tabular}

Heart rate decreased in the isoproterenol group $(P<0.01)$. No significant change was documented in HR in groups P20, P40 and P80 compared to isoproterenol.

After receiving isoproterenol, LVEDP increased by more than five times $(P<0.001)$, which 4-PBA could diminish this change in $P 20, P 40$, and $P 80$ groups $(P<0.01, P<0.001$, and $P<0.01$, respectively).

Isoproterenol group had lower $L V d P / d t_{\max }$ and $L V d P / d t_{\text {min }}$ than the control group $(P<0.01, P<0.05$ respectively). $P 20$ increased both the max and min LV dP/dt $(P<0.01, P<0.05$ respectively). $P 40$, only made significant increase in $L V$ $\mathrm{dP} / \mathrm{dtmax}(\mathrm{P}<0.05)$. In contrast, max and min LV dP/dt were not effectively changed by P80, compared to isoproterenol. The results showed that the changes trend in LV dP/dt/P were similar to the changes in min LV dP/dt.

\section{Effect of 4-PBA on the heart weight/body weight ratio}

To assess cardiac hypertrophy and edema, the ratio of the heart weight (in grams) to the rat body weight (in kilograms) was calculated and demonstrated in figure 1. The heart weight/body weight ratio increased in the isoproterenol group $(\mathrm{P}<0.01)$. 4-PBA in none of the treated groups could remarkably influence this ratio compared to isoproterenol $(P>0.05)$.

\section{Effect of 4-PBA on cardiac tissue histopathology}

As shown in figure 2, in the control group, myocardial fibers were arranged regularly with distinctive striation. No clear degeneration or necrosis was noted. Histological sections of cardiac tissue in the isoproterenol group showed extensive subendocardial necrosis, hypertrophy, abundant fibroblastic hyperplasia, and capillary dilation and leukocyte infiltration. P40 with less amount of these isoproterenol-induced injuries, significantly decreased the inflammatory response and destruction $(\mathrm{P}<0.001)$. In contrast, changes in groups $\mathrm{P} 20$ and P80 were not significant $(P>0.05)$. To report the quantitative analysis of histological results, grading of histopathological changes of heart tissue after isoproterenol injection and in the presence of different doses of 4-PBA are also shown in figure 2.

Effect of 4-PBA on the peripheral blood neutrophils and serum TAC 
The peripheral blood neutrophils count and serum TAC are demonstrated in figure 3. Following the injection of isoproterenol and induction of inflammation, the percentage of peripheral blood neutrophils in the isoproterenol group was significantly augmented compared to the control group $(P<0.001)$. This ratio decreased in all 4-PBA receiving groups with a significant difference in $P 40(P<0.01)$ and $P 80(P<0.001)$.

The serum TAC significantly decreased in the isoproterenol group compared with the control group $(P<0.001)$. Using the $80 \mathrm{mg} / \mathrm{kg}$ of 4-PBA, made a notable serum TAC augmentation ( $\mathrm{P}<0.001$ versus isoproterenol group). $\mathrm{P} 20$ and $\mathrm{P} 40$ had not considerable TAC variation ( $P>0.05)$.

\section{Results of western blotting}

The Beclin1/GAPDH protein ratio increased in the isoproterenol group compared to the control. An insignificant change in P20, P40 and P80 groups was documented for Beclin1/GAPDH compared to the isoproterenol group $(P>0.05$, Figure 4).

The difference in P62/GAPDH protein level between the isoproterenol and the healthy control groups was not remarkable $(\mathrm{P}>0.05)$. However, the P40 and P80 significantly decreased the P62 expression compared to the isoproterenol group $(P<0.05$, Figure 5$)$.

The ratio of LC3-II/LC3-I/GAPDH protein was insignificantly higher in the isoproterenol group compared to the control $(P>0.05)$. In groups receiving 4-PBA, this amount decreased in the P20 group and again increased in the P40 and P80 groups compared with the isoproterenol group, however none of these changes were statistically significant $(P>0.05$, Figure 6).

\section{Discussion}

This study assessed the cardiac pharmacological effects of 4-PBA in rats with isoproterenol-induced MI. The hypothesis was that injection of 4-PBA by interfering in autophagy pathways would improve the pathological status of the heart. The results showed that the cardiac injury caused by isoproterenol significantly decreased following the administration of 4-PBA.

Isoproterenol is a beta-agonist sympathetic stimulant that induces $\mathrm{Ml}$, changes the hemodynamic parameters, and causes necrosis, fibrosis, inflammation, and neutrophil accumulation [38]. An increase in incompetent proteins level occurs in oxidative stress and MI, which result in further damage [39]. The cardiac status after MI may be improved by potential of 4-PBA in the regulation of oxidative stress and elimination of unfolded and misfolded proteins [40].

In this study, administration of 4-PBA in dosage of 20 and $40 \mathrm{mg} / \mathrm{kg}$ significantly improved the hemodynamic parameters. They reversed all the heart-weakening effects of isoproterenol by increasing the MAP, LV dP/dt/P, LV $\mathrm{dP} / \mathrm{dt}_{\mathrm{max}}, \mathrm{LV} \mathrm{dP} / \mathrm{dt}_{\mathrm{min}}$, and LVSP and decreasing the LVEDP. Considering the LV dp/dt/P results, the mechanism of 4PBA to demonstrate these hemodynamic influences is highly by enhancing the cardiac muscle power and less likely through changing the volume or preload. In contrast, the variation in hemodynamic parameters was not significant following the injection of $80 \mathrm{mg} / \mathrm{kg}$ of 4-PBA. This controversy can be due to excessive stimulation of autophagy by the higher dose of 4-PBA and consequent elimination of essential proteins of cells, which could induce apoptosis in cardiac cells $[41,42]$.

Administration of 4-PBA in concentration of $40 \mathrm{mg} / \mathrm{kg}$ decreased necrosis, fibrosis, and inflammation caused by isoproterenol in cardiac tissue. A previous research on the effect of 4-PBA on cardiac necrosis and fibrosis indicated that injection of $80 \mathrm{mg} / \mathrm{kg}$ 4-PBA in $1 \mathrm{~h}$ before induction of $\mathrm{Ml}$, decreased fibrosis and necrosis in rats by minimizing 
the oxidative stress [31]. Another study assessed the efficacy of 4-PBA to prevent the effects of thapsigargin on cardiac fibroblasts isolated from rats. It showed that 4-PBA decreased the endoplasmic reticulum stress and accumulation of procollagen in cardiac fibroblasts and consequently decreased the cell death [43]. Our results were in line with their findings with the difference that $40 \mathrm{mg} / \mathrm{kg}$ of 4-PBA in our study corresponded to approximately 80 $\mathrm{mg} / \mathrm{kg}$ dosage of 4-PBA sodium salt in previous studies.

Administration of 40 and $80 \mathrm{mg} / \mathrm{kg}$ of 4 -PBA had striking effect in reducing the percentage of peripheral blood neutrophils, while this fluctuation was not significant following the injection of $20 \mathrm{mg} / \mathrm{kg}$ of 4 -PBA. This finding can be due to the demonstrating effective anti-inflammatory role in higher doses of 4-PBA, which have been previously reported. A study performed to assessment of the efficacy of 4-PBA in palliation of inflammation caused by dextran sulfate sodium (DSS) in mice colon. It exerted anti-inflammatory effects in higher dose of $150 \mathrm{mg} / \mathrm{kg}$ by suppressing

the nuclear factor-kB activation and inhibiting the pro-inflammatory cytokines, interleukin-6, interleukin-1B, and tumor necrosis factor-alpha [44].

Compatible with neutrophils count abatement, the increase in serum TAC was only significant in high dose of 80 $\mathrm{mg} / \mathrm{kg}$ of 4-PBA. This result can be related to the declining of oxidative stress, which result in increasing the antioxidant capacity and decreasing cell damages [45].

The ratio of heart weight/body weight significantly increased following the injection of isoproterenol compared with the control group. This increase, seems to be due to the edema and cardiac tissue inflammation, and less likely related to cardiac tissue hypertrophy. As we assessed acute $\mathrm{MI}$ and there was not sufficient time for the cardiac hypertrophy occurrence. Injection of 4-PBA did not cause a significant change in this ratio, which indicates inefficacy of 4-PBA for tissue edema.

The results of western-blotting tests showed that the level of P62 significantly decreased following the 40 and 80 $\mathrm{mg} / \mathrm{kg}$ of 4-PBA, indicating the induction of the macro-autophagy pathway. In addition, the variation trend of other proteins in this cascade were also towards the induction of macro-autophagy, though not significant. There are different reports introducing the 4-PBA as a chemical chaperon with potential of inducing the CMA pathway. Considering the correlation of CMA with the macro-autophagy pathways, by induction of CMA, the macro-autophagy pathway is suppressed [46-48]. In this study, in contrast to our expectations, injection of 4-PBA induced the macroautophagy pathway. This may be due to a sudden increase in unfolded or misfolded proteins in $\mathrm{MI}$, which can activate both macro-autophagy and CMA pathways at the same time [49]. Moreover, P62 can serve as a mediator between the CMA and macro-autophagy pathways, suggesting the cross-talking of these two pathways [50]. The obtaining sinus rhythm in effective results of different dosages of 4-PBA can show the fact that altering the autophagy and CMA are so dose-dependent and in special optimum concentration of 4-PBA can occur.

Furthermore, differences in effective doses can be due to differences in the targets of this medication. Tissue and hemodynamic changes probably show different patterns in response to administration of 4-PBA. Future studies with deep focus on CMA pathways and markers are required on the cardiac functions of 4-PBA.

\section{Conclusion}

The current results showed that injection of 4-PBA caused significant improvements in hemodynamic factors, histopathological changes, inflammation, serum TAC, and neutrophil accumulation, which demonstrated the cardioprotective effects in isoproterenol-induced MI. As the autophagy modulation potential of 4-PBA, understanding its 
compensatory mechanisms between the different autophagic pathways is important in light of the development of interventions aimed for therapeutic purposes with altering autophagy especially in cardiac disorders.

\section{Declarations}

FundingThis work was supported by the vice chancellor for research of Tabriz University of medical sciences. It belonged to study design number 60013 , which was confirmed by the ethical committee of Tabriz medical university (IR.TBZMED.VCR.REC.1397.048).

Competing Interests The authors have no relevant financial or non-financial interests to disclose.

Author contributions All authors contributed to the study conception and design. Fatemeh Vatankhah performed most of the experiments and drafted the manuscript; Haleh Vaez designed, conceived the experiments and analyzed the study and corrected the manuscript; Alireza Garjani supervised the study and corrected the manuscript.

Ethics approval This study was conducted under the institutional guidelines for the care and use of animal models. The approvement by the ethics committee of Tabriz University of Medical Sciences obtained (IR.TBZMED.VCR.REC.1397.139 and IR.TBZMED.VCR.REC.1397.048).

Availability of data and material (data transparency) The data that support the findings of this study are available from the corresponding author, [Haleh Vaez], upon reasonable request.

\section{References}

1. Shepard D, VanderZanden A, Moran A, Naghavi M, Murray C, Roth G. Ischemic Heart Disease Worldwide, 1990 to 2013: Estimates From the Global Burden of Disease Study 2013. Circ Cardiovasc Qual Outcomes. 2015;8(4):4556.

2. Frangogiannis NG, Smith CW, Entman ML. The inflammatory response in myocardial infarction. Cardiovasc Res. 2002;53(1):31-47.

3. Thorp EB. The Myocardial Unfolded Protein Response during Ischemic Cardiovascular Disease. Biochem Res Int. 2012;2012:583170.

4. Thuerauf DJ, Marcinko M, Gude N, Rubio M, Sussman MA, Glembotski CC. Activation of the unfolded protein response in infarcted mouse heart and hypoxic cultured cardiac myocytes. Circ Res. 2006;99(3):275-82.

5. Levine B, Klionsky DJ. Development by self-digestion: molecular mechanisms and biological functions of autophagy. Dev Cell. 2004;6(4):463-77.

6. Gozuacik D, Kimchi A. Autophagy and cell death. Curr Top Dev Biol. 2007;78:217-45.

7. Mizushima N. A brief history of autophagy from cell biology to physiology and disease. Nat Cell Biol. 2018;20(5):521-7.

8. Klionsky DJ, Emr SD. Autophagy as a regulated pathway of cellular degradation. Science. 2000;290(5497):171721.

9. Yu L, Chen Y, Tooze SA. Autophagy pathway: Cellular and molecular mechanisms. Autophagy. 2018;14(2):20715.

10. Feng Y, He D, Yao Z, Klionsky DJ. The machinery of macroautophagy. Cell Research. 2014;24(1):24-41.

11. Arias E, Cuervo AM. Chaperone-mediated autophagy in protein quality control. Curr Opin Cell Biol. 2011;23(2):184-9. 
12. Wu H, Chen S, Ammar A-B, Xu J, Wu Q, Pan K, et al. Crosstalk Between Macroautophagy and ChaperoneMediated Autophagy: Implications for the Treatment of Neurological Diseases. Molecular Neurobiology. 2015;52(3):1284-96.

13. Ott C, König J, Höhn A, Jung T, Grune TJRB. Macroautophagy is impaired in old murine brain tissue as well as in senescent human fibroblasts. 2016;10:266 - 73.

14. Kang R, Zeh HJ, Lotze MT, Tang D. The Beclin 1 network regulates autophagy and apoptosis. Cell Death Differ. 2011;18(4):571-80.

15. Rusten TE, Stenmark H. p62, an autophagy hero or culprit? Nature Cell Biology. 2010;12(3):207-9.

16. Komatsu M, Ichimura Y. Physiological significance of selective degradation of p62 by autophagy. FEBS Lett. 2010;584(7):1374-8.

17. Komatsu M, Waguri S, Koike M, Sou YS, Ueno T, Hara T, et al. Homeostatic levels of p62 control cytoplasmic inclusion body formation in autophagy-deficient mice. Cell. 2007;131(6):1149-63.

18. Bjørkøy G, Lamark T, Pankiv S, Øvervatn A, Brech A, Johansen T. Monitoring autophagic degradation of p62/SQSTM1. Methods Enzymol. 2009;452:181-97.

19. Massey AC, Kaushik S, Sovak G, Kiffin R, Cuervo AM. Consequences of the selective blockage of chaperonemediated autophagy. Proc Natl Acad Sci U S A. 2006;103(15):5805-10.

20. Kaushik S, Massey AC, Mizushima N, Cuervo AM. Constitutive Activation of Chaperone-mediated Autophagy in Cells with Impaired Macroautophagy. 2008;19(5):2179-92.

21. Tanida I, Ueno T, Kominami E. LC3 and Autophagy. Methods Mol Biol. 2008;445:77-88.

22. Tanida I, Minematsu-Ikeguchi N, Ueno T, Kominami E. Lysosomal turnover, but not a cellular level, of endogenous LC3 is a marker for autophagy. Autophagy. 2005;1(2):84-91.

23. Taylor SC, Berkelman T, Yadav G, Hammond M. A defined methodology for reliable quantification of Western blot data. Mol Biotechnol. 2013;55(3):217-26.

24. Galluzzi L, Bravo-San Pedro JM, Levine B, Green DR, Kroemer G. Pharmacological modulation of autophagy: therapeutic potential and persisting obstacles. Nature reviews Drug discovery. 2017;16(7):487-511.

25. Cortez L, Sim V. The therapeutic potential of chemical chaperones in protein folding diseases. Prion. 2014;8(2):197-202.

26. Perlmutter DH. Chemical chaperones: a pharmacological strategy for disorders of protein folding and trafficking. Pediatr Res. 2002;52(6):832-6.

27. Mimori S, Ohtaka H, Koshikawa Y, Kawada K, Kaneko M, Okuma Y, et al. 4-Phenylbutyric acid protects against neuronal cell death by primarily acting as a chemical chaperone rather than histone deacetylase inhibitor. Bioorg Med Chem Lett. 2013;23(21):6015-8.

28. Kim SW, Hooker JM, Otto N, Win K, Muench L, Shea C, et al. Whole-body pharmacokinetics of HDAC inhibitor drugs, butyric acid, valproic acid and 4-phenylbutyric acid measured with carbon-11 labeled analogs by PET. Nucl Med Biol. 2013;40(7):912-8.

29. PETER T. 4-phenylbutyric acid controlled-release formulations for therapeutic use. LUNAMED INC (CH) TRUOG PETER (CH); 2006.

30. Park CS, Cha H, Kwon EJ, Sreenivasaiah PK, Kim DH. The chemical chaperone 4-phenylbutyric acid attenuates pressure-overload cardiac hypertrophy by alleviating endoplasmic reticulum stress. Biochem Biophys Res Commun. 2012;421(3):578-84. 
31. Ayala P, Montenegro J, Vivar R, Letelier A, Urroz PA, Copaja M, et al. Attenuation of endoplasmic reticulum stress using the chemical chaperone 4-phenylbutyric acid prevents cardiac fibrosis induced by isoproterenol. Exp Mol Pathol. 2012;92(1):97-104.

32. Kilkenny C, Browne WJ, Cuthill IC, Emerson M, Altman DG. Improving bioscience research reporting: the ARRIVE guidelines for reporting animal research. PLoS Biol. 2010;8(6):e1000412.

33. Luo ZF, Feng B, Mu J, Qi W, Zeng W, Guo YH, et al. Effects of 4-phenylbutyric acid on the process and development of diabetic nephropathy induced in rats by streptozotocin: regulation of endoplasmic reticulum stress-oxidative activation. Toxicol Appl Pharmacol. 2010;246(1-2):49-57.

34. Garjani A, Andalib S, Biabani S, Soraya H, Doustar Y, Garjani A, et al. Combined atorvastatin and coenzyme Q10 improve the left ventricular function in isoproterenol-induced heart failure in rat. Eur J Pharmacol. 2011;666(13):135-41.

35. Yousefi K, Soraya H, Fathiazad F, Khorrami A, Hamedeyazdan S, Maleki-Dizaji N, et al. Cardioprotective effect of methanolic extract of Marrubium vulgare L. on isoproterenol-induced acute myocardial infarction in rats. Indian J Exp Biol. 2013;51(8):653-60.

36. Benjamin IJ, Jalil JE, Tan LB, Cho K, Weber KT, Clark WA. Isoproterenol-induced myocardial fibrosis in relation to myocyte necrosis. Circulation research. 1989;65(3):657-70.

37. Benzie IF, Strain JJ. The ferric reducing ability of plasma (FRAP) as a measure of "antioxidant power": the FRAP assay. Anal Biochem. 1996;239(1):70-6.

38. Upaganlawar A, Gandhi H, Balaraman R. Isoproterenol Induced Myocardial Infarction: Protective Role of Natural Products. Journal of Pharmacology and Toxicology. 2011;6(1):1-17.

39. Benjamin IJ, McMillan DR. Stress (heat shock) proteins: molecular chaperones in cardiovascular biology and disease. Circ Res. 1998;83(2):117-32.

40. Ringe D, Petsko GA. What are pharmacological chaperones and why are they interesting? J Biol. 2009;8(9):80.

41. Martinet W, Agostinis P, Vanhoecke B, Dewaele M, De Meyer GR. Autophagy in disease: a double-edged sword with therapeutic potential. Clin Sci (Lond). 2009;116(9):697-712.

42. Shintani T, Klionsky DJ. Autophagy in health and disease: a double-edged sword. Science. 2004;306(5698):9905.

43. Humeres C, Montenegro J, Varela M, Ayala P, Vivar R, Letelier A, et al. 4-Phenylbutyric acid prevent cytotoxicity induced by thapsigargin in rat cardiac fibroblast. Toxicol In Vitro. 2014;28(8):1443-8.

44. Ono K, Nimura S, Nishinakagawa T, Hideshima Y, Enjyoji M, Nabeshima K, et al. Sodium 4-phenylbutyrate suppresses the development of dextran sulfate sodium-induced colitis in mice. Exp Ther Med. 2014;7(3):573-8.

45. Zhou SX, Zhou Y, Zhang YL, Lei J, Wang JF. Antioxidant probucol attenuates myocardial oxidative stress and collagen expressions in post-myocardial infarction rats. J Cardiovasc Pharmacol. 2009;54(2):154-62.

46. Massey AC, Kaushik S, Sovak G, Kiffin R, Cuervo AM. Consequences of the selective blockage of chaperonemediated autophagy. Proceedings of the National Academy of Sciences of the United States of America. 2006;103(15):5805-10.

47. Park H-J, Son H-J, Sul O-J, Suh J-H, Choi H-S. 4-Phenylbutyric acid protects against lipopolysaccharide-induced bone loss by modulating autophagy in osteoclasts. Biochemical pharmacology. 2018;151:9-17.

48. Kim DS, Li B, Rhew KY, Oh HW, Lim HD, Lee W, et al. The regulatory mechanism of 4-phenylbutyric acid against ER stress-induced autophagy in human gingival fibroblasts. Arch Pharm Res. 2012;35(7):1269-78. 
49. Liang S, Ping Z, Ge J. Coenzyme Q10 Regulates Antioxidative Stress and Autophagy in Acute Myocardial Ischemia-Reperfusion Injury. Oxid Med Cell Longev. 2017;2017:9863181.

50. Wu H, Chen S, Ammar AB, Xu J, Wu Q, Pan K, et al. Crosstalk Between Macroautophagy and Chaperone-Mediated Autophagy: Implications for the Treatment of Neurological Diseases. Mol Neurobiol. 2015;52(3):1284-96.

\section{Figures}

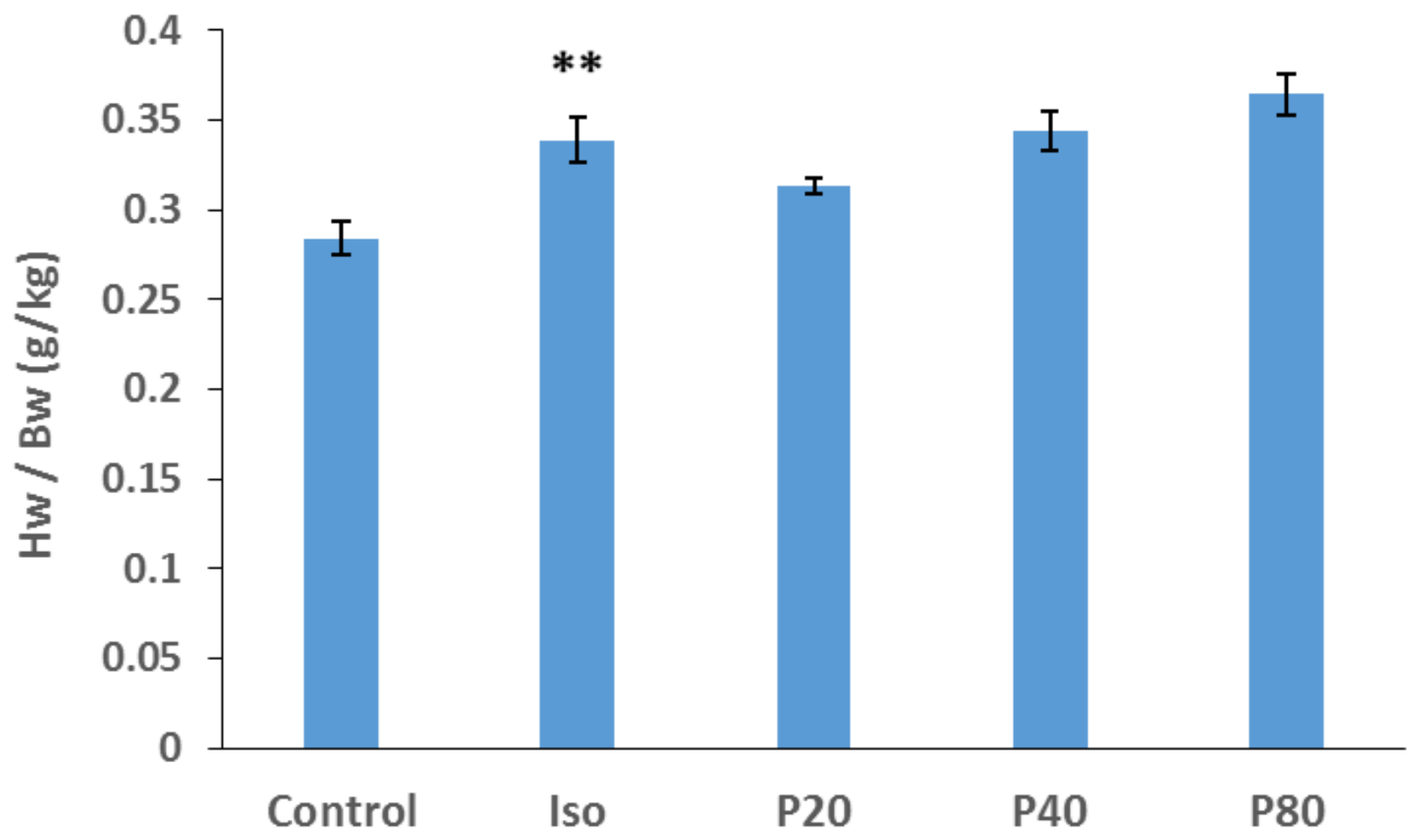

Figure 1

Effects of 4-PBA on heart weight/body weight ratio. The data were expressed as mean \pm SEM $(n=6)$. The ratio of the heart weight (HW, in grams) to the rat body weight (BW, in kilograms) was calculated. Iso: isoproterenol; P20: 4-PBA $(20 \mathrm{mg} / \mathrm{kg})+$ isoproterenol; P40: 4-PBA $(40 \mathrm{mg} / \mathrm{kg})+$ isoproterenol; P80: 4-PBA $(80 \mathrm{mg} / \mathrm{kg})+$ isoproterenol. ${ }^{* *} \mathrm{P}<0.01$ from control group using ANOVA and LSD post-hoc test. 
Control



Iso+ P20



Iso+P80

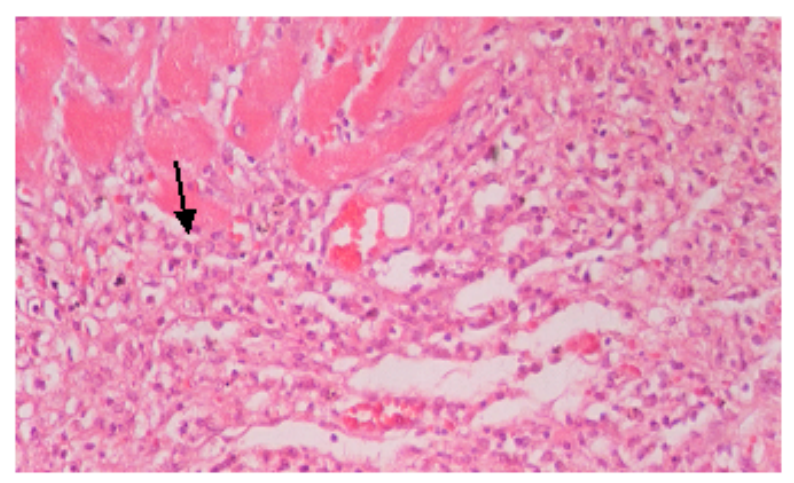

Iso

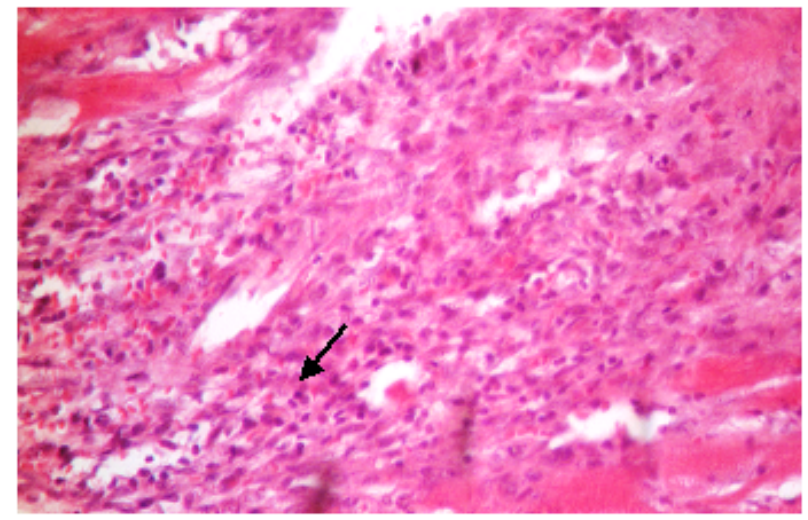

Iso $+\mathrm{P} 40$
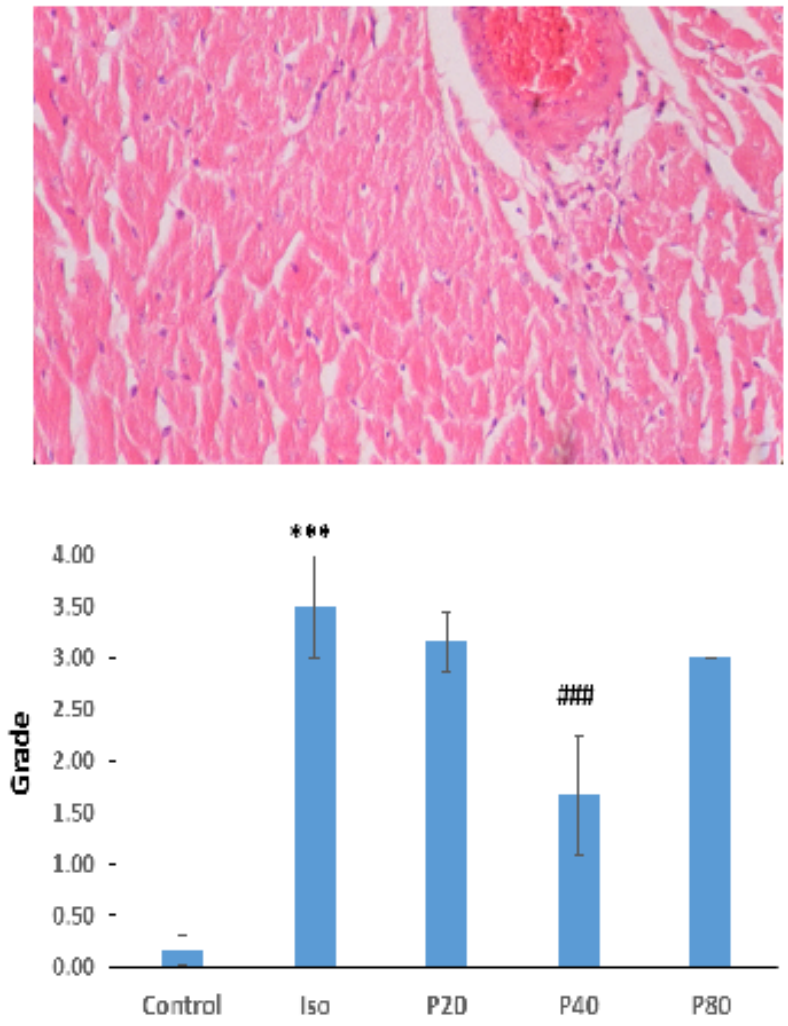

\section{Figure 2}

Photomicrographs of sections of rat cardiac apexes for evaluating necrosis, tissue disorientation and neutrophil accumulation. The rat cardiac tissue in the isoproterenol group showed fibrosis, cardiomyocyte necrosis, and local infiltration of mononuclear cells (black arrows). Treatment with $40 \mathrm{mg} / \mathrm{kg}$ 4-PBA caused a significant improvement. Sectioned at five $\mu \mathrm{m}$ and stained with Hematoxylin and Eosin (H\&E) (4OM). Quantitative analysis was performed through grading of histopathological changes. Results presented as mean \pm SEM $(n=5)$. Iso: isoproterenol; P20: 4PBA (20 mg/kg) + isoproterenol; P40: 4-PBA (40 mg/kg) + isoproterenol; P80: 4-PBA (80 mg/kg) + isoproterenol.

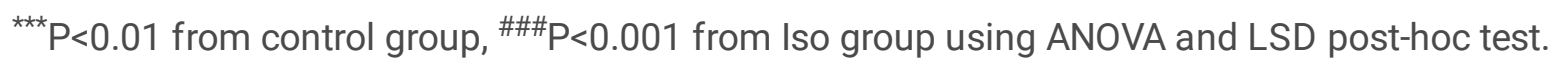




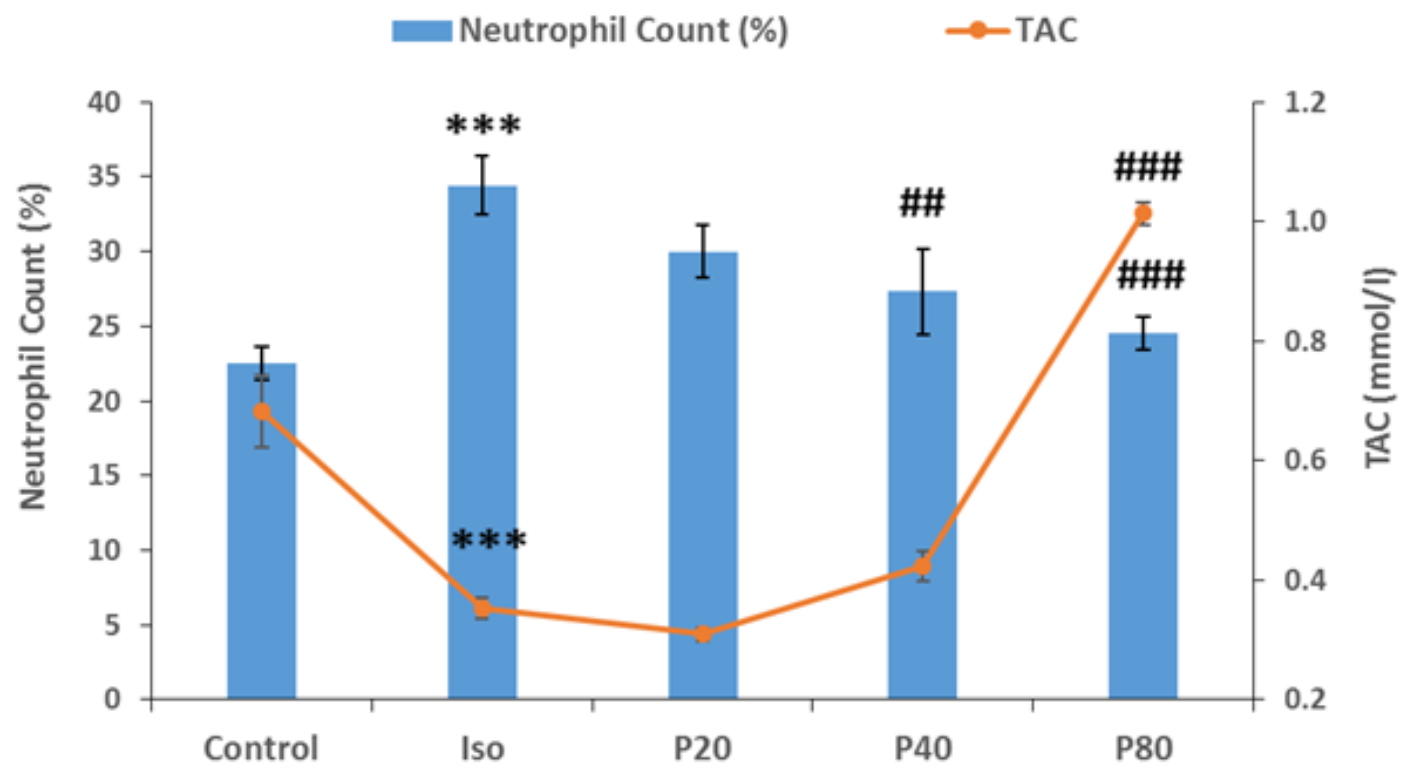

Figure 3

Effects of 4-PBA on percentage of peripheral blood neutrophils. The data were expressed as mean \pm SEM $(n=6)$. Iso: isoproterenol; P20: 4-PBA (20mg/kg) + isoproterenol; P40: 4-PBA (40 mg/kg) + isoproterenol; P80: 4-PBA (80 mg/kg) + isoproterenol. ${ }^{* \star *} \mathrm{P}<0.01$ from control group, ${ }^{\# \#} \mathrm{P}<0.01,{ }^{\# \# \#} \mathrm{P}<0.001$ from Iso group using ANOVA and LSD post-hoc test.

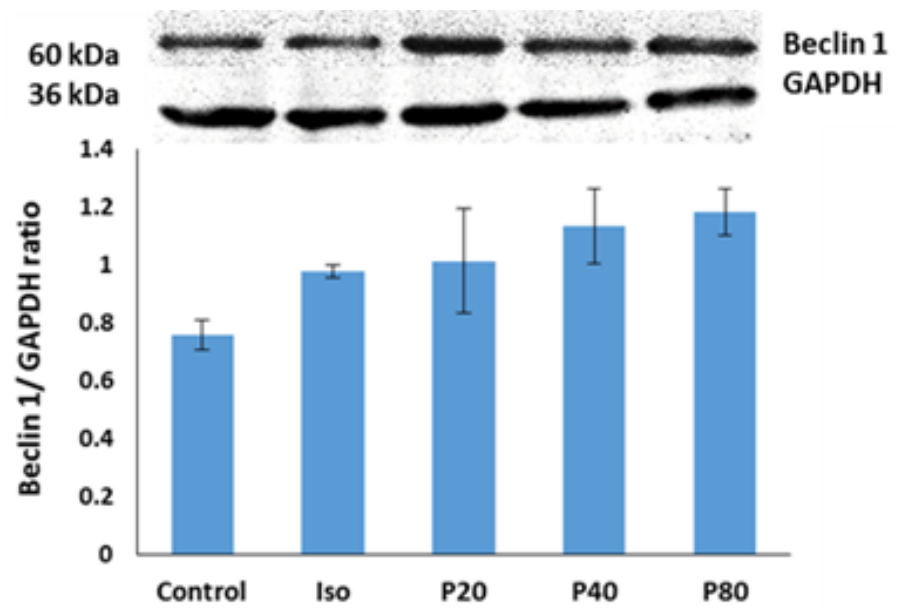

Figure 4

Effect of 4-PBA on Beclin1/GAPDH ratio. The data were expressed as mean \pm SEM $(n=4)$. Iso: isoproterenol; $P 20$ : 4PBA (20mg/kg) + isoproterenol; P40: 4-PBA (40 mg/kg) + isoproterenol; P80: 4-PBA (80 mg/kg) + isoproterenol. The samples derive from the same experiment or parallel experiments and that gels/blots were processed in parallel. 


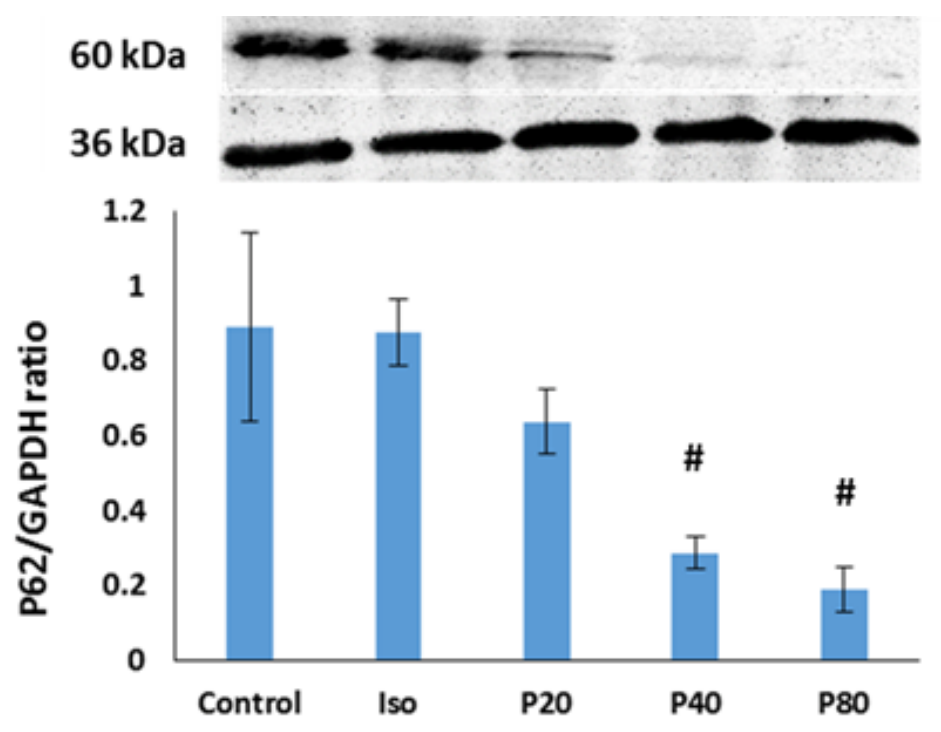

Figure 5

Effect of 4-PBA on P62/GAPDH ratio. The data were expressed as mean \pm SEM $(n=4)$. Iso: isoproterenol; P20: 4-PBA $(20 \mathrm{mg} / \mathrm{kg})+$ isoproterenol; P40: 4-PBA $(40 \mathrm{mg} / \mathrm{kg})+$ isoproterenol; P80: 4-PBA $(80 \mathrm{mg} / \mathrm{kg})+$ isoproterenol. ${ }^{\mathrm{P}}<0.05$ from isoproterenol treated group using ANOVA and LSD post-hoc test. The samples derive from the same experiment or parallel experiments and that gels/blots were processed in parallel.

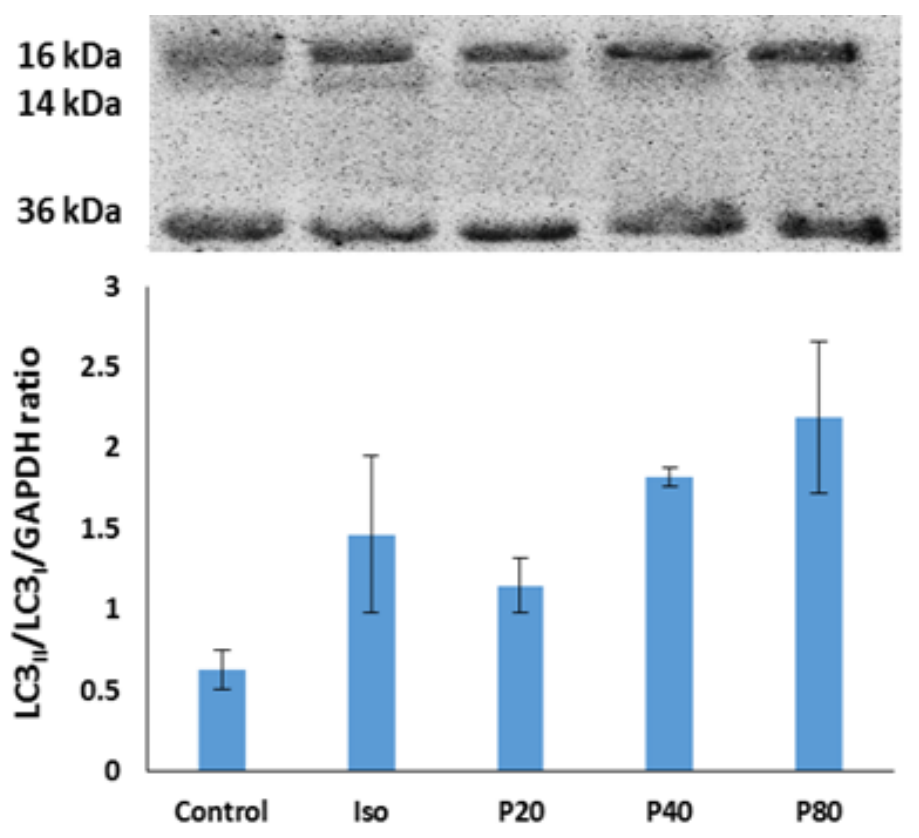

LC3

LC3,

GAPDH

\section{Figure 6}

Effect of 4-PBA on LC3-II/LC3-I/GAPDH. The data were expressed as mean \pm SEM $(n=4)$. Iso: isoproterenol; P20: 4PBA (20 mg/kg) + isoproterenol; P40: 4-PBA (40 mg/kg) + isoproterenol; P80: 4-PBA (80 mg/kg) + isoproterenol. The samples derive from the same experiment or parallel experiments and that gels/blots were processed in parallel. 


\section{Supplementary Files}

This is a list of supplementary files associated with this preprint. Click to download.

- Graphicalabstract.tif 